\title{
Cerebral oedema in diabetic ketoacidosis
}

\author{
Still puzzling-and often fatal
}

Despite improvements in insulin delivery and monitoring of fluid and electrolyte balance the mortality associated with diabetic ketoacidosis has remained largely unchanged for the past 25 years at about $10 \%$. One of the principal causes of death is cerebral oedema, a largely unexplained complication of the ketoacidotic state with a mortality of over $90 \%$. The incidence of this complication is particularly high in children. Over $95 \%$ of cases occur in those aged under 20 years, with a third under the age of 5 years'; and as many as a tenth of children with diabetic ketoacidosis may have subclinical cerebral oedema. ${ }^{2}$

Dillon et al first described cerebral oedema as a common finding in uncomplicated fatal diabetic acidosis in $1936 .{ }^{3}$ By the 1960 s it had become evident that, far from being prevented by treatment, cerebral oedema developed after the start of appropriate management and might result from it. Subsequent studies, however, have failed to implicate any single aspect of management.

The rate of rehydration might be expected to have a role in the genesis of cerebral oedema since a rapid fall in plasma osmolality would favour net transport of water into cells. A rate of administration of fluid of greater than $4 \mathrm{l} / \mathrm{m}^{2} /$ day has been argued to predispose patients to cerebral oedema, ${ }^{+}$yet one study has found that nearly half the cases occurred in patients who had inadequate fluid replacement. ${ }^{1}$ Both glucose and sodium contribute to the osmolality in ketoacidosis, and concentrations can rapidly change during treatment. But in over $40 \%$ of reported cases of cerebral oedema the fall in blood glucose concentration was less than $2.8 \mathrm{mmol} / \mathrm{l} / \mathrm{h}$. ${ }^{1}$ The association with disturbances in sodium balance is stronger: a third of patients had a plasma sodium concentration of less than $130 \mathrm{mmol} / \mathrm{l}$ at the time of cerebral herniation ${ }^{4}$ and almost $60 \%$ had a fall in concentration of over $4 \mathrm{mmol} / 1 .^{1} \mathrm{~A}$ combination of changes related to treatment may conspire to lower plasma osmolality precipitately, thus expanding the intracellular fluid volume. There is no evidence that other aspects of management, such as giving bicarbonate or potassium, are relevant.

If therapeutic practices are not directly responsible for cerebral oedema what other mechanisms may be important? The two principal hypotheses are the generation of osmoprotective molecules in brain cells during the onset of ketoacidosis and impaired ion transport.

When hyperglycaemia is induced in animals the cerebrospinal fluid and brain extracellular space become hyperosmolar, which is only partly accounted for by an increase in glucose concentration. Clements et al showed an accumulation of sorbitol and fructose in the cerebrospinal fluid in hyperglycaemic dogs and found that when saline was infused to lower blood glucose concentration rapidly sorbitol continued to accumulate with an associated rise in cerebrospinal fluid pressure. ${ }^{5}$ Cerebral oedema was found at necropsy in those animals with the largest increases in cerebrospinal fluid pressure. They thus proposed that metabolism of glucose in brain cells through the polyol pathway caused accumulation of osmotically active molecules such as sorbitol and myoinositol, leading to intracerebral hyperosmolality. Arieff and Kleeman, however, found no significant change in the concentration of these molecules in the brain extracellular space in hyperglycaemic rabbits. ${ }^{6}$ They attributed half the increase in brain osmolality to the accumulation of unidentified osmoprotective "idiogenic osmoles," which prevented shrinkage of brain cells in the face of the plasma hyperosmolality in diabetic ketoacidosis. Rapid correction of hyperglycaemia with insulin caused further accumulation of these molecules, and brain osmolality was maintained as plasma osmality fell, thus precipitating cerebral oedema. When the hyperglycaemia was corrected by peritoneal dialysis cerebral oedema did not occur, implying that insulin treatment itself may help to cause the oedema. However, the blood glucose concentration in these experiments fell by 11-18 $\mathrm{mmol} / \mathrm{l} / \mathrm{h}$, which is more rapid than usual, and the animals were not acidotic, so these models would seem to parallel more closely the hyperosmolar non-ketotic state.

A potential mechanism is activation of the sodiumhydrogen exchanger, which would lead to cytoplasmic accumulation of sodium. But in models of diabetic ketoacidosis sodium does not accumulate in brain cells and cerebral $\mathrm{pH}$ is not greatly decreased, so weak organic acids probably do not enter brain cells to cause cytoplasmic acidification.

The excitatory amino acid receptors, such as the $n$-methyl-D-aspartate (NMDA) receptor, have recently been implicated in the generation of cerebral oedema in ischaemia. Young and Bradley have suggested that in diabetic ketoacidosis tissue hypoxia may be responsible for the development of cerebral oedema. ${ }^{7}$ Cerebral hypoxia leads to release of glutamate, causing toxic activation of the NMDA receptor. This results in excitotoxic cell swelling as a result of ion influx and a toxic increase in intracellular calcium. ${ }^{8}$

Thus a combination of rapid changes in plasma osmolality, 
osmoprotective molecules in brain cells, and altered membrane ion transport, possibly as a result of hypoxia, is probably responsible for the development of cerebral oedema in diabetic ketoacidosis.

Though the aetiology of the condition is still unclear, management must aim to minimise the risk. Cerebral oedema usually presents 2-24 hours after treatment has started, with abrupt neurological deterioration and coma followed by respiratory arrest. In retrospect warning signs, such as headache, incontinence, and behavioural changes are often present a few hours earlier. The only conventional treatment that may help is mannitol. This is most effective when given soon after the onset of oedema, with over half the reported patients left with mild or no disability. ${ }^{19}$ The only danger is of plasma hyperosmolality after repeated doses. Dexamethasone does not seem to help and hyperventilation may be harmful since the fall in arterial carbon dioxide concentration will reduce cerebral perfusion pressure, predisposing to ischaemic damage.

Finally, in view of the probable role of changes in plasma osmolality, abrupt changes in glucose and electrolyte concentrations should be avoided. Colloid and crystalloid replacement could be alternated during initial rehydration to maintain plasma osmolality while the fluid and electrolyte abnormalities are corrected. When possible, plasma osmo- lality and concentrations of blood glucose and plasma sodium should be monitored and the rates of insulin and saline infusion adjusted to avoid rapid reduction; a fall in osmolality of less than $5 \mathrm{mmol} / \mathrm{h}$ is optimal. By such intensive monitoring it may be possible to reduce the incidence of this devastating complication.

PETER HAMMOND MRC Training Fellow

SIMON WALLIS W Senior Lecturer

Division of Endocrinology and Metabolism, Royal Postgraduate Medical School, London W12 0NN

1 Rosenbloom AL. Intracerebral crises during treatment of diabetic ketoacidosis. Diabetes Care 1990;13:1-32.

2 Krane EJ, Rockoff MA, Wallman JK, Wolfsdorf JI. Subclinical brain swelling in children during treatment of diabetic ketoacidosis. $N$ Engl f Med 1985;312;1147-51.

3 Dillon ES, Riggs HE, Dyer WW. Cerebral lesions in uncomplicated fatal diabetic acidosis. Am ₹ Med Sci 1936;192:360-5.

4 Duck SC, Wyatt DT. Factors associated with brain herniation in the treatment of diabetic ketoacidosis. $\mathcal{F}$ Pediatr 1988;113:10-4.

5 Clements RS, Prockop LD, Winegrad AI. Acute cerebral oedema during the treatment of hyperglycaemia: an experimental model. Lancet 1968;ii:384-6.

6 Arieff AI, Kleeman CR. Effects of hyperglycaemia and rapid lowering of plasma glucose in normal rabbits. F Clin Invest 1973;52:571-83.

Young E, Bradley RF. Cerebral oedema with irreversible coma in severe diabetic ketoacidosis. $N$ Engl f Med 1967;276:665-9.

8 Collins RC, Dobkin BH, Choi DW. Selective vulnerability of the brain: new insights into the pathophysiology of stroke. Ann Intern Med 1989;110:992-1000.

9 Bello FA, Sotos JF. Cerebral oedema in diabetic ketoacidosis in children. Lancet 1990;336:64.

\section{Assisted conception on the NHS?}

\section{Many infertile couples could be helped}

Assisted conception, which includes the use of in vitro fertilisation, gamete intrafallopian transfer, and intrauterine insemination, is unusual in many respects. Very few techniques have been introduced into medical practice and developed under the same degree of public and professional scrutiny. Test tube babies raised political, ethical, and social questions such that it is the only branch of British medicine whose practice is highly regulated by statute law. ${ }^{1}$ With few exceptions health authorities have refused to fund assisted conception within the NHS so that its development and delivery have been almost entirely in the private sector. The dearth of well conducted trials of clinical efficacy is therefore unsurprising, although the registration of results through the Voluntary Licensing Authority, Interim Licensing Authority, and now the Human Fertilisation and Embryology Authority has provided a national audit of activity and results. $^{2}$

Steptoe and Edwards tried varying techniques for more than 10 years before the birth of the first baby after in vitro fertilisation in $1978 .{ }^{3}$ Initially the success rates were very low, but they have improved since then owing to several factors. It was soon realised that the pregnancy rate per cycle increased in proportion to the number of embryos transferred, although with it the risk of multiple pregnancy increased. ${ }^{4}$ Current regimens of ovarian stimulation with gonadotrophins after down regulation with agonists of gonadotrophin releasing hormone have resulted in fewer cancelled cycles due to premature discharge of luteinising hormone. ${ }^{5}$ Moreover, it has allowed the recovery of the eggs at times suitable to both woman and doctor. Refinements of the conditions of embryo culture, better instrumentation, and probably a less stressful environment have also contributed to improved results such that many centres are now restricting to two the number of embryos replaced in favourable couples. ${ }^{6}$ The remaining $\stackrel{\circ}{\circ}$ unused embryos may be frozen and used in subsequent $\cong$ cycles.

Little doubt therefore exists that in vitro fertilisation and other forms of assisted conception offer a chance of treatment to some infertile couples who otherwise would remain childless. Two recently published studies suggest that in selected patients cumulative pregnancy rates are similar to those in normal fertile couples. Hull et al found that this was 3 true for couples where the woman was under 40 and the man had normal sperm. ${ }^{8}$ Reviewing the results of in vitro fertilisation for all causes of infertility, Tan et al found that womeno under 34 had comparable pregnancy rates to the normal population. After 35, pregnancy and birth rates fell rapidly. ${ }^{\circ} \rightarrow$ The success rate falls in older women because of difficulty in stimulating multiple follicular development, a reduced implantation rate per embryo, and a higher proportion of N spontaneous miscarriages. All these factors point to fewer $\sigma$ normal oocytes and more embryos with less developmental

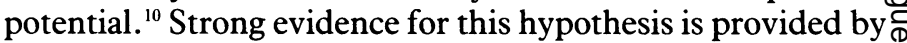
the fact that when donor oocytes are used the pregnancy and? live birth rate for recipient older women is very high."

Why do governments and the World Health Organisation ${ }^{12} \frac{\mathrm{O}}{8}$ have such reservations about a successful treatment for women who would otherwise remain infertile? Some hold the view that such expensive treatment should be accorded a low priority when compared with life saving procedures such aso renal transplantation. WHO points out the risks of the hyperstimulation syndrome and the increased incidence of $\frac{\bar{\sigma}}{\partial}$ abortion and preterm labour associated with multiple preg-nancies, which further drain the limited funds available for health. Analysis of more than 1000 babies born after in vitro fertilisation, however, shows an increase in preterm deliv- 